\title{
Determining multiallelic complex copy number and sequence variation from high coverage exome sequencing data
}

\author{
Diego Forni ${ }^{1,2}$, Diana Martin ${ }^{1}$, Razan Abujaber ${ }^{1}$, Andrew J. Sharp ${ }^{3}$, Manuela Sironi ${ }^{2}$ and Edward J. Hollox ${ }^{1 *}$
}

\begin{abstract}
Background: Copy number variation (CNV) is a major component of genomic variation, yet methods to accurately type genomic CNV lag behind methods that type single nucleotide variation. High-throughput sequencing can contribute to these methods by using sequence read depth, which takes the number of reads that map to a given part of the reference genome as a proxy for copy number of that region, and compares across samples. Furthermore, high-throughput sequencing also provides information on the sequence differences between copies within and between individuals.

Methods: In this study we use high-coverage phase 3 exome sequences of the 1000 Genomes project to infer diploid copy number of the beta-defensin genomic region, a well-studied CNV that carries several beta-defensin genes involved in the antimicrobial response, signalling, and fertility. We also use these data to call sequence variants, a particular challenge given the multicopy nature of the region.

Results: We confidently call copy number and sequence variation of the beta-defensin genes on 1285 samples from 26 global populations, validate copy number using Nanostring nCounter and triplex paralogue ratio test data. We use the copy number calls to verify the genomic extent of the CNV and validate sequence calls using analysis of cloned PCR products. We identify novel variation, mostly individually rare, predicted to alter amino-acid sequence in the betadefensin genes. Such novel variants may alter antimicrobial properties or have off-target receptor interactions, and may contribute to individuality in immunological response and fertility.
\end{abstract}

Conclusions: Given that $81 \%$ of identified sequence variants were not previously in dbSNP, we show that sequence variation in multiallelic CNVs represent an unappreciated source of genomic diversity.

Keywords: Exome, High throughput sequencing, Copy number variation, Beta-defensin

\section{Background}

Copy number variation $(\mathrm{CNV})$, where a section of DNA differs from a diploid copy number of 2 between different individuals, is a common form of variation that can affect a substantial portion of the human genome and genomes from other organisms [1-3]. Such variation can affect phenotype through a variety of mechanisms, such as a gene dosage effect [4], variation in the number of protein coding domains [5] or alteration of position of enhancer elements [6]. CNV can be divided into simple CNV, usually comprising a deletion or duplication and generated by one mutational event, and complex multiallelic $\mathrm{CNV}$ where different

\footnotetext{
* Correspondence: ejh33@le.ac.uk

'Department of Genetics, University of Leicester, Leicester, UK

Full list of author information is available at the end of the article
}

dosage variants are generated by recurrent mutation. In humans, complex $\mathrm{CNV}$ has been shown to affect disease susceptibility. For example, CNV of the beta-defensin locus has been shown to affect the risk of developing the inflammatory skin disease psoriasis $[7,8]$, and CNV of the neuronal glucose transporter SLC2A3 modifies the age of onset of Huntington's disease [9]. Furthermore, CNV of the amylase gene in dogs has been shown to be an adaptation to domestication and in Drosophila CNV is important in resistance to insecticides $[10,11]$.

Despite the importance of complex CNV, research progress has lagged behind studies into simpler deletions and duplications, and has been dogged by false positive disease associations. This is almost entirely due to the challenges in accurately typing complex multiallelic CNV [12]. Most 
studies have used real-time quantitative PCR, which is prone to bias $[13,14]$. Although other more reliable methods exist, such as the paralogue ratio test (PRT) and multiplex amplifiable probe hybridisation (MAPH), such assays require extensive validation and are locus specific rather than genomewide, limiting the amount of data generated at one time. Genome-wide approaches, such as analysis of hybridisation signal intensities from SNP chips and array comparative genomic hybridisation, generate a large amount of useful data but are often weak in typing complex $\mathrm{CNV}$ - i.e. giving an absolute copy number rather than just indicating a loss or gain of signal. Furthermore, complex CNV detection and typing appears to be DNA cohort dependent, which further increases the risk of false negative genetic associations and false positive associations due to batch effects.

Recent work has addressed analysis of CNVs using sequence read depth (SRD) generated by next-generation sequencing platforms $[4,15]$. The principle is simple: when short read sequences are generated from an individual with a high copy number for a particular region and are subsequently aligned to a reference genome with only one copy of that region, the number of reads mapping to that region will be higher than expected. Most studies have focused on detecting CNV in whole genome sequences rather than typing absolute copy number of particular complex CNV regions [16]. One exception relies on singly-unique-nucleotides (SUNs) to provide an internal calibration of copy number of that particular region [15]. This has been successful for some CNVs, and using this approach the role of a particular sequence variant of the CNV carrying the SRGAP2 gene in human evolution has been elucidated [17]. Nevertheless, the extent to which other complex CNVs show that particular pattern of variation, where SUNs exist and are frequent, is not clear.

One distinct advantage of SRD approaches is that they can yield information on the sequence differences between different copies of the complex CNV. Other methods, such as analysis of SNP array hybridisation intensity data, array comparative genomic hybridisation $(\mathrm{aCGH})$ data, $\mathrm{MAPH}$ or PRT often assume sequence identity under the probe or primer. The effect of this can be minimised either by multiple assays or careful design of probes/assays, but such methods remain essentially blind to the sequence variation between copies. This sequence variation can be important, for example, in the SRGAP2 example mentioned above, where only particular paralogues, defined by sequence variants, are functional [17].

An accurate assessment of sequence variation of a multiallelic CNV locus using SRD needs high coverage sequence data to be confident that all copies have their sequence represented in the alignment. The number of publicallyavailable whole genomes sequenced at high coverage is increasing but limited, but the 1000 Genome Project has released sequence data of 2455 exomes sequenced at high coverage using Illumina sequencing technology. Furthermore, given the lower cost of exome sequencing per sample, exome sequences are becoming increasingly common for large sample sets. Therefore we focused on complex CNV typing and sequence analysis using high coverage exome sequence data.

There are several platforms that use exome SRD data to detect and call CNV [16]. All use a similar approach of using principal component analysis to extract components describing different aspects of the variation, including noise, and then using a hidden Markov model approach to call boundaries between gain of copy number, loss of copy number and diploid regions. In principle, the variation in the principal component that most closely corresponds to DNA dosage signal can be used to type the absolute integer copy number of complex CNV. Only one platform has been used to do this, CoNIFER, which has been used on 907 exomes to show proof of principle [18], and subsequently on 1644 exomes to investigate the role of $\mathrm{CNV}$ in autism spectrum disorder [19].

To rigorously test the quality of copy number and sequence variation calls from exome data on a region of complex CNV, we decided to focus on the well-studied human beta-defensin locus. This is a $322 \mathrm{~kb}$ region of DNA on chromosome 8p23.1, which is embedded within a complex region rich in segmental duplications and olfactory receptor genes $[20,21]$. The $322 \mathrm{~kb}$ region is copy number variable as a block, with the diploid copy number commonly between 2 and 7 copies, but individuals with as few as one copy and as many as 12 copies have been observed [14, 22]. Chromosomes with high copy number of the beta-defensin region can be distinguished by a visibly larger $8 \mathrm{p} 23.1$ region by G-band staining [23]. Furthermore, the beta-defensin region is polymorphic in physical location on the chromosome, with some copies polymorphically at the proximal end of $8 \mathrm{p} 23.1$ within the olfactory repeat region REPP in addition to the distal end within the olfactory repeat region REPD [20].

Seventeen annotated genes are within the copy number variable region, including eight beta-defensin genes (DEFB4, DEFB103, DEFB104, DEFB105, DEFB106, $D E F B 107, D E F B 108$ and $D E F B 109)$ and nine other genes mostly expressed in the testes [21]. The role of $D E F B 4$ and $D E F B 103$ appear to be immunomodulatory in humans, in addition to direct antimicrobial activity. For the other defensins, the role is less clear, although a knockout mouse deleting the orthologous beta-defensin cluster shows complete male infertility, strongly suggesting an important role of these genes in reproduction [22]. The function of the other genes remains unknown. Sequence variation of the region remains understudied, primarily due to the difficulty of reliably distinguishing variant nucleotides in multicopy regions by the Sanger sequencing method. We therefore decided to test whether 
exome SRD could accurately call copy number and sequence variation of this complex CNV, both to identify beta-defensin variants which may have novel functions but also to robustly establish a recommended approach that could be applied to complex CNVs by the research community.

\section{Methods}

\section{Exome data}

Filtered phase 3 exome data generated by the 1000 Genome project was downloaded from the European Bioinformatics Institute (http://ftp.1000genomes.ebi.ac.uk/ vol1/) as fastq files. We retrieved only Illumina technology filtered paired end data generated by four different sequencing centres: Baylor College of Medicine (BCM), Beijing Genomic Institute (BGI), Broad Institute (BI), and Washington University Genome Sequencing Center (WUGSC). The initial dataset consisted of 2455 samples divided in 5 major continental groups (referred to as "super populations" by the 1000 Genomes Project): African, East Asian, South Asian, European, and Admixed American. Throughout this paper, the standard abbreviations for 1000 Genomes populations are used - see http:// www.1000genomes.org/category/frequently-asked-questions/ population for details of population codes and sample names.

\section{Mapping}

The reference sequence was generated using all chromosome 8 exons plus 300 bp flanking sequence from the GRCh37-hg19 human reference genome; when two exons were closer than $600 \mathrm{bp}$, all the genomic distance was considered. We used mrsFAST ultra v3.2.0 for sequence alignment, using a paired end approach [24]. Importantly, ConIFER was designed to be used on alignments generated using mrsFAST [25], a one-to many sequence read aligner, rather than other more common aligners based on the Burrows-Wheeler algorithm that generally operate as a one-to-one sequence aligner. mrsFAST therefore captures all possible mapping locations up to a user-defined number of mismatches, which leads to more accurate estimates of copy number from SRD approaches, because all copies above a given percentage identity will be aligned efficiently to the reference locus. A reference indexing window size of 12 was created and the pair end reads mapping was performed using the all-mapper tool with a maximum error threshold of 6 .

\section{CNV calling}

CNV calling used CoNIFER, a suite of Python scripts that calculate RPKM (reads per thousand bases per million reads sequenced) values starting from aligned sequences and a set of probes [18]; for our analyses we defined the probe set as all the chr8 exon boundaries. Samples with a RPKM mean $<50$ were discarded from the analysis to reduce background signal. Singular value decomposition standardized z-scores RPKM (SVD-ZRPKM) values were calculated for samples belonging to one or more population generated by the same sequencing centre (e.g. all African samples from BGI), removing the first components that are disproportionally responsible for the variance of the data. For each dataset under analysis, we evaluated the best number of components to be removed using scree plots generated by CoNIFER; the components to be removed were chosen based on the shoulder of the scree plot. Raw SVD-ZRPKM mean of the beta-defensins

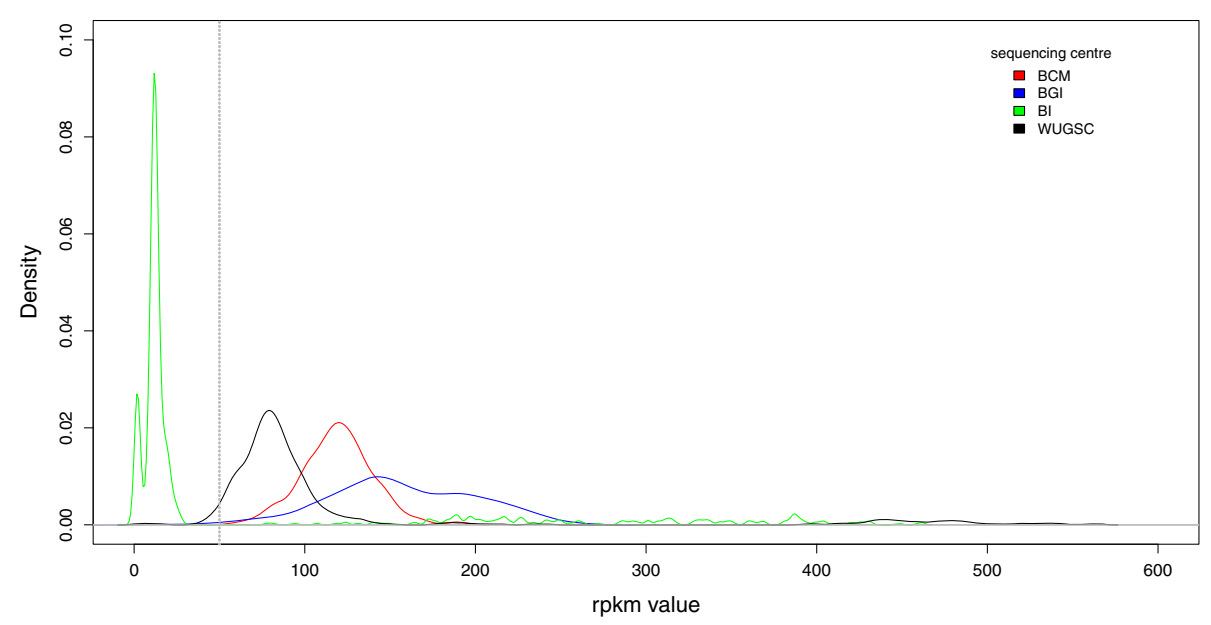

Fig. 1 Distribution of reads-per-kilobase-per-million-reads (RPKM) values of different samples stratified by sequencing centre. The kernel density plot shows density of RPKM values from mrsFAST alignments for four different sequencing centres, distinguished by the different colours. The vertical dotted line indicates the cutoff value at RPKM $=50$, with samples above that threshold taken on for copy number calling 
that unambiguously map just to chromosome $8 \mathrm{p} 23.1$ DEFB4, DEFB103, DEFB104, DEFB105, DEFB106 and $D E F B 107)$ were retrieved and used for CNV calling. To determine the integer copy number call for each sample we used CNVtools, grouping samples from the same continental group and sequencing centre together. The Gaussian mixture models were evaluated by eye, plotting the mixture model together with a histogram of raw copy number to check for clear clustering about integer copy numbers, and also by comparing calls with previous copy number estimates of samples from the HapMap collection that overlapped the 1000 Genomes dataset, using triplex paralogue ratio tests [26]. These positive controls also allowed us to confirm that the correct SVD-ZRPKM component was used for copy number calling.

\section{Variant calling}

Single nucleotide variants in one assembled beta-defensin region were called using FreeBayes v9.9.2 [27]. Freebayes is a haplotype-based Bayesian genetic variant detector, which can call sequence variants from samples of different ploidy, with ploidy (equivalent to, in our case, copy number) as an extra parameter for each sample. We ran FreeBayes setting 30 and 50 as minimum base and mapping quality, respectively; 0.10 as the minimum fraction of alternate variant observations and 10 for the minimum count of alternate variant reads; finally we specified for each sample the number of copies of the beta-defensin region estimated by the CoNIFER exome SRD analysis.

\section{Validation of copy number}

Copy number estimates of 164 samples from the HapMap collection that overlapped the 1000 Genomes dataset, using triplex paralogue ratio tests, have been published previously. Copy number estimates of the same samples were also obtained using the Nanostring nCounter system [28], by designing 6 probes mapping to the beta-defensin repeat and calculating the first principal component, sample-wise, of the 6 probe values using the $R$ package CNVtools [29].

\section{Validation of sequence variants}

PCR primers were designed flanking variable sites, and genomic DNA from a selection of 8 HapMap samples amplified using standard PCR. PCR products were then cloned into pJET 1.2 vector using the CloneJet PCR cloning kit (Thermo Scientific), where the insert disrupts a lethal gene allowing only plasmids with an insert to confer viability to the bacterial host. Following plating, colonies were selected for colony PCR using the vector primers 5'CGA CTCACTATAGGGAGAGCGGC-3' and 5'AAGAACATCGATTTTCCATGGCAG-3' flanking the insert, according to the manufacturer's instructions. PCR products were then digested using the appropriate restriction enzyme to distinguish the alleles at the variant site (MspI for rs140952426, ApeKI for rs200757797) and scored for allelic state following agarose gel electrophoresis.

\section{Population genetics}

Variant frequency distributions for non-synonymous and synonymous variants were compared across the betadefensin genes analysed, for each population, using the k-sample Anderson-Darling test [30] implemented by the $\mathrm{R}$ package kSamples.

\section{Availability of data}

One thousand Genomes phase 3 exome read data are publically available from the European Bioinformatics Institute European Bioinformatics Institute (http:// $\mathrm{ftp} .1000$ genomes.ebi.ac.uk/vol1/) as fastq files. All 1285 confident copy number calls have been submitted to dbVar (http://www.ncbi.nlm.nih.gov/dbvar) with accession number nstd116. All novel variants identified in this paper have been deposited in dbSNP (http:// www.ncbi.nlm.nih.gov/SNP/).

\section{Ethics statement}

For a description of the ethics and consent agreements used for the 1000 Genomes Project see http:// www.1000genomes.org/about.

Table 1 Characteristics of exome sequences analysed

\begin{tabular}{llllll}
\hline Centre name & Samples & $\begin{array}{l}\text { Samples with }>50 \text { reads } \\
\text { per exon }\end{array}$ & $\begin{array}{l}\text { Sample with a copy } \\
\text { number call }\end{array}$ & $\begin{array}{l}\text { Samples with a copy number } \\
\text { call } P>0.95^{*}\end{array}$ & $\begin{array}{l}\text { Sequence enrichment } \\
\text { method }\end{array}$ \\
\hline BCM & 228 & 228 & 147 & $129(88 \%)$ & HSGC VCRome custom array \\
BGI & 894 & 866 & 866 & $795(91 \%)$ & $\begin{array}{l}\text { NimbleGen } \\
\text { SeqCap_EZ_Exome V2 }\end{array}$ \\
BI & 830 & 161 & 161 & $140(87 \%)$ & $\begin{array}{l}\text { Agilent } \\
\text { SureSelect_All_Exon_V2 }\end{array}$ \\
WUGSC & 503 & 397 & 288 & $221(76 \%)$ & $\begin{array}{l}\text { NimbleGen } \\
\text { SeqCap_EZ_Exome v3 }\end{array}$ \\
\end{tabular}




\section{Results}

We aligned fastq raw sequence read files for 2455 samples from 26 populations to a concatenated reference sequence made of all chromosome 8 exons plus 300 bp flanking sequence. We grouped the samples from the 26 populations into four continental groups. After removing samples where the reads per kilobase per million mapped reads (RPKM) mean value was less than 50, (Fig. 1, Table 1) we calculated

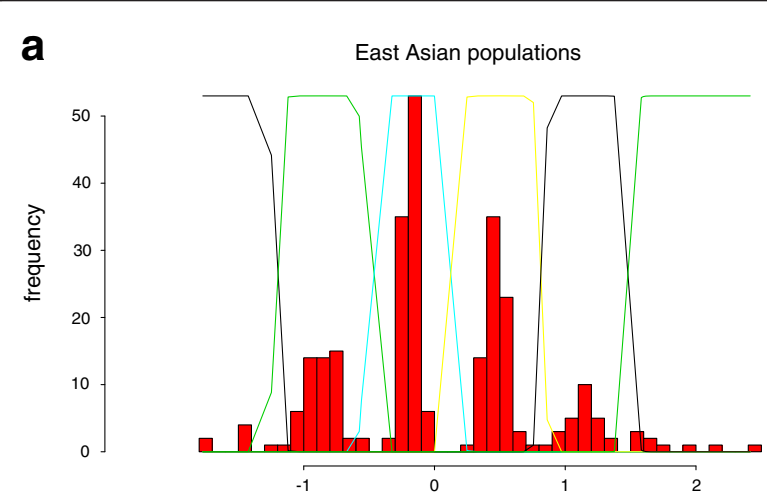

b

South Asian populations

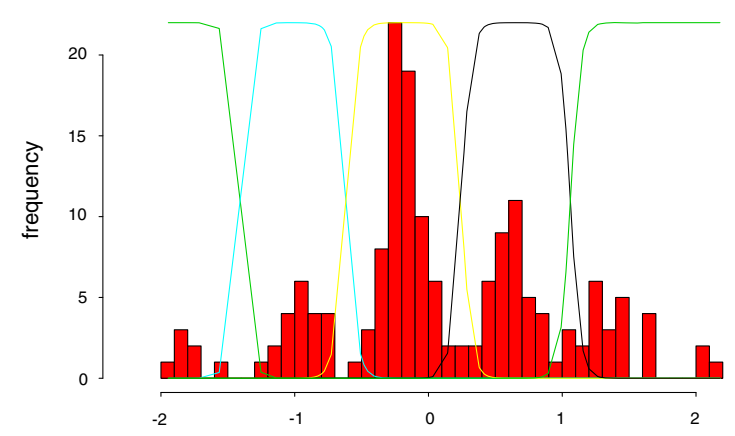

C

South Asian and East Asian populations

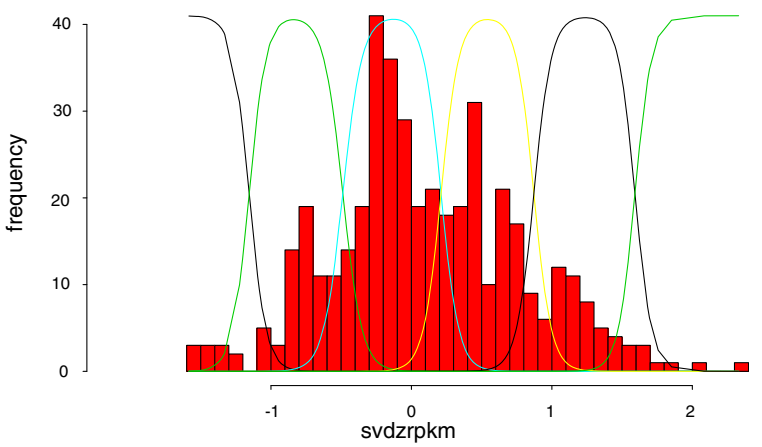

Fig. 2 Effects of continental group batch of origin on copy number clustering. The histograms show normalised sequence depth coverage data for the beta-defensin region generated by the BGl sequencing centre. $X$-axis values represent raw mean SVD-ZRPKM values, and the $y$-axis represents number of samples. Curved lines indicating the Gaussian curves used to call integer copy number. a) samples from East Asian populations $(n=269)$, b) samples from South Asian populations $(n=165), \mathbf{c})$ samples from South Asian and East Asian populations analysed as one batch $(n=434)$ single variant decomposition scores batch wise, with each batch representing a distinct continental group/sequencing centre combination. Raw SVD-ZRPKM mean of all exons of the beta-defensin genes were retrieved and a Gaussian mixture model fitted for each set of data using CNVtools. Observing clear clustering of SVD-ZRPKM mean values about integer copy number values leads us to have high confidence in the Gaussian mixture model fit and therefore in the final copy number calls. Importantly, batches needed to be defined by both sequencing centre and continental group, otherwise poor clustering was observed. For example, Fig. 2 shows histograms of raw SVD-ZRPKM mean values from the BGI sequencing centre, for East Asians (Fig. 2a), South Asians (Fig. 2b) and East and South Asians together (Fig. 2c). Although a Gaussian mixture model can be fitted for all three histograms, the clustering of the SVDZRPKM values of the combined batches (Fig. 2c) is visibly less distinct than when each batch is analysed separately. For most batches, clear clustering of raw SVD-ZRPKM values was observed, increasing confidence that the correct copy number was being called (Fig. 3a). However some showed SVD-ZRPKM values that did not cluster well (e.g. African samples from BCM sequencing centre, Fig. 3b), and these were removed from subsequent analyses.

Gaussian mixture modelling generated a copy number call for each sample with an associated posterior probability of that call. The proportion of calls with a posterior probability greater than 0.95 varied between sequencing centres (Table 1), but overall was $87 \%$. The distribution of copy number reflected previous results, with 4 being the modal copy number in all continental groups apart from sub-Saharan Africans, and the range of common variation extending from 2 copies to 8 copies per diploid genome (Table 2).

We validated our copy number calls by comparing calls on a subset of samples with copy number estimates made previously by Triplex PRT $[13,26]$, by Nanostring [28] (Fig. 4a), and also with copy number calls of the region made by whole genome sequencing [4] (Fig. 4b). It is clear that copy number calls made using exome SRD agree well with both PRT and Nanostring consensus copy number. Exome SRD calls also agree well with whole genome SRD data, although there is a significant discrepancy rate of $11.8 \%$. Most discrepancies are at the higher copy numbers, and seem to be due to exome SRD underestimating copy number. Of the 7 samples that are discrepant between exome SRD and both PRT and Nanostring, three are also discrepant with whole genome SRD copy number calls, all are spread across the different sequencing centres (Table 3), suggesting the discrepancies are due to random assay noise rather than a systematic bias.

We used our exome SRD data to investigate the extent of contiguous copy number variation at this locus, gene 

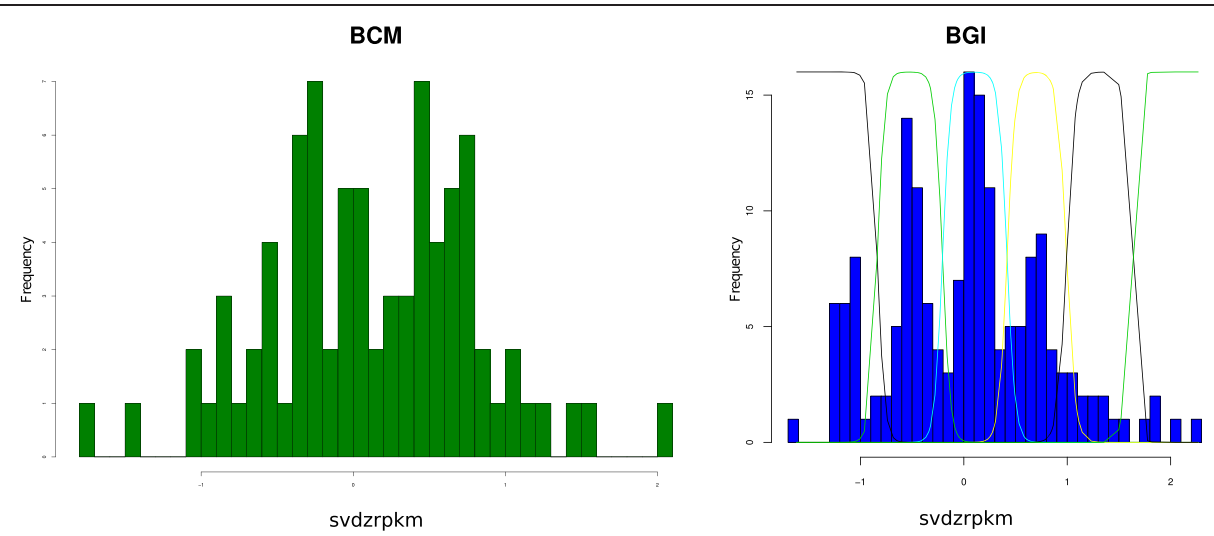

Fig. 3 Effects of sequencing centre and batch size on copy number clustering. The histograms show normalised sequence depth coverage data for the beta-defensin region for sub-Saharan African samples. X-axis values represent raw mean SVD-ZRPKM values a) BCM sequencing centre, $n=81$ (15 YRI, 57 LWK, 9 ASW). b) BGl sequencing centre, $n=172$ (26 YRI, 3 LWK, 25 GWD, 43 MSL, 47 ESN, 5 ASW, 23 ACB), with curved lines indicating the Gaussian mixture model used to call integer copy number

by gene. Individual SVD-ZRPKM mean values of each gene were correlated with the individual SVD-ZRPKM mean values of genes within and surrounding the betadefensin $\mathrm{CNV}$ region, both at distal 8p23.1 (a region called REPD [31]), and proximal 8p23.1 (REPP), across the 171 European samples sequenced at the BGI. We would expect genes on the CNV block to show highly correlated SVD-ZRPKM scores across these individuals, reflecting the $\mathrm{CNV}$. Indeed, the core defensin genes (DEFB4 to DEFB107) showed a very high correlation (Fig. 5) indicating that these are on a contiguous block that shows CNV. This block of highly correlated genes extends distally as far as FAM90A13 and includes DEFB109, albeit with lower correlation coefficients, which is likely to be due to mapping of sequence reads derived from known segmental duplications involving these genes on chromosome 4 and chromosome 12 . This confirms the observation made previously using arrayCGH that these genes are involved in the beta-defensin CNV [21], and shows that analysis of exome SRD can be a powerful approach to identify $\mathrm{CNV}$ boundaries. Interestingly, a moderate correlation coefficient is observed for some genes at REPP, including DEFB130 but not DEFB134, DEFB135 nor DEFB136. The beta-defensin repeat region (involving DEFB4 to FAM90A13) is not assembled here, but it is known from genetic data that the repeat region can be polymorphically present here at this location [20], and this signal we observe is likely to be due to CNV of the beta-defensin repeat region at REPP.

We used our exome alignment files to call sequence variation across the beta-defensin genes within the $\mathrm{CNV}$. Using FreeBayes, a sequence caller that uses diploid copy number as an extra parameter and therefore can make sequence variant calls from non-diploid regions, we called 436 single nucleotide variants spanning 8811 bp of sequence representing the combined length of the beta-defensin genes. 299 are intronic or intergenic, with 137 within the untranslated regions or coding regions. The majority of variants called are rare or very rare, and are specific to particular continental groups, suggesting that they have arisen very recently in human evolutionary history. 355 variants ( $81 \%$ of total) are novel and have been submitted to dbSNP.

Sixty-seven variants (64 non-synonymous substitutions and 3 stop codon gains) were called that were predicted to affect amino acids within the beta-defensin genes

Table 2 Beta-defensin copy number frequency in human continental groups

\begin{tabular}{lllllllll}
\hline Continental group & Total Samples & 2 copy & 3 copy & 4 copy & 5 copy & 6 copy & 7 copy & 8 copy \\
\hline Sub-Saharan African & 214 & $1(0.005)$ & $26(0.12)$ & $60(0.28)$ & $68(0.32)$ & $38(0.18)$ & $16(0.07)$ & $5(0.02)$ \\
East Asian & 405 & $13(0.03)$ & $81(0.200)$ & $153(0.38)$ & $112(0.28)$ & $34(0.08)$ & $10(0.02)$ & $2(0.005)$ \\
South Asian & 248 & $9(0.04)$ & $41(0.17)$ & $97(0.39)$ & $66(0.27)$ & $32(0.13)$ & $3(0.01)$ & 0 \\
European & 349 & $7(0.02)$ & $50(0.14)$ & $142(0.41)$ & $109(0.31)$ & $35(0.10)$ & $6(0.02)$ & 0 \\
Admixed American & 246 & $6(0.02)$ & $49(0.20)$ & $113(0.46)$ & $64(0.26)$ & $12(0.05)$ & $2(0.008)$ & 0 \\
\hline
\end{tabular}




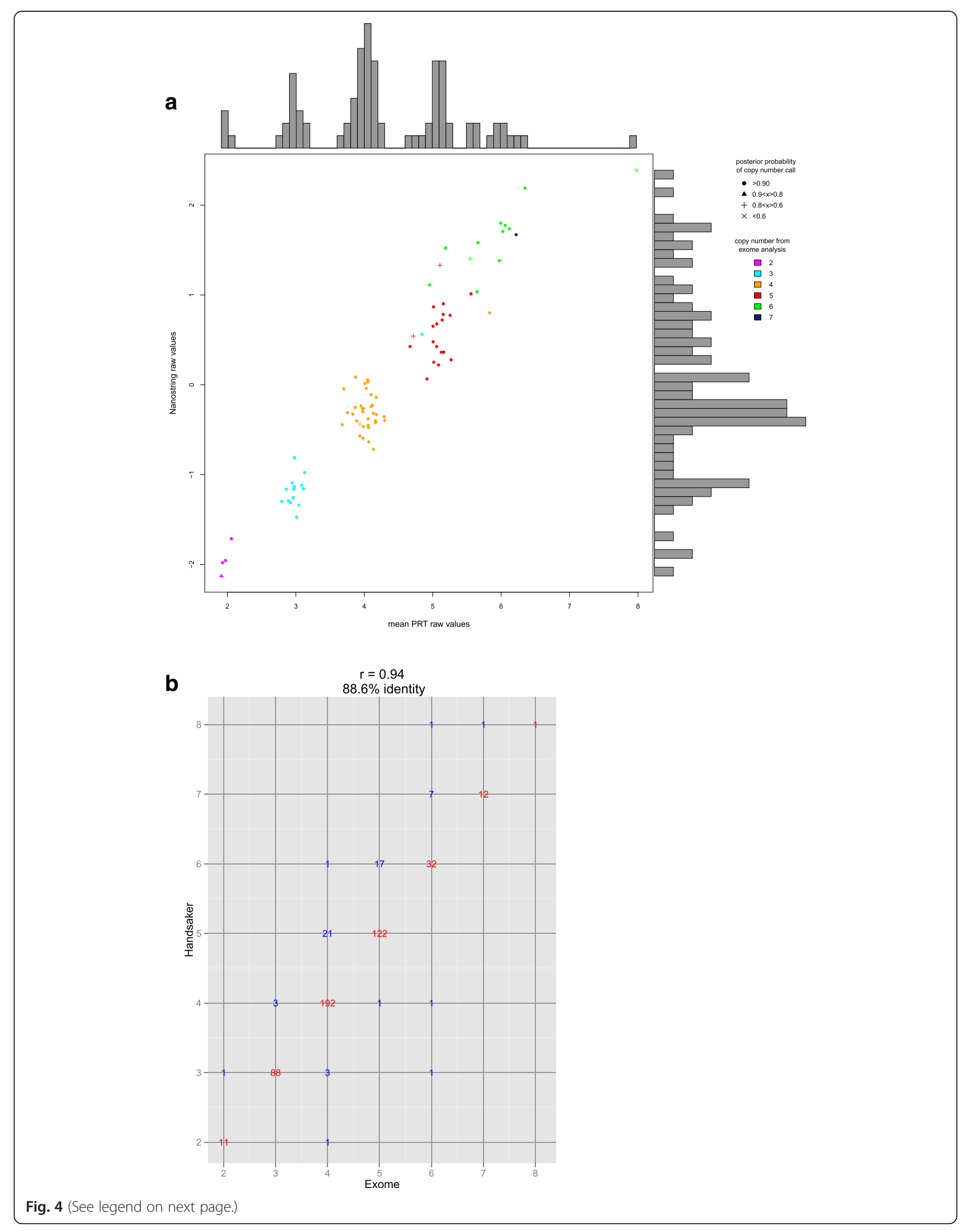


(See figure on previous page.)

Fig. 4 Validated of beta-defensin copy number calling. The plots show comparisons between two methods of calling integer beta-defensin copy number. a) comparison with triplex paralogue ratio test and Nanostring nCounter. b) comparison with integer calls from phase 1 low coverage whole genome data [4]. The figures in red indicate the numbers of samples concordant for that particular copy number. The numbers in blue indicate the numbers of discordant samples

(Fig. 6). We validated two frequent non-synonymous variants, rs140952426 in DEFB104 that changes arginine to a glutamine at position 38, and rs200757797 in DEFB105 that changes a cysteine to a tyrosine at position 73. It was important not only to validate the presence of the variant but also the correct number of copies of that variant. We did this by amplifying across the variant using genomic DNA, cloning the resulting PCR product, and then counting the number of clones (each derived from a single amplified DNA molecule from the PCR) that had each allele of the variant using colony PCR followed by restriction enzyme digestion (Table 4). This gave an estimate of the proportion of each allele at each variant for each sample, which could be then compared with that predicted by exome sequencing - for example a GGGA genotype (where three copies have a $\mathrm{G}$ and one copy has an $\mathrm{A}$ at the same paralogous nucleotide site) would be regarded as $0.25 \mathrm{~A}$ allele. For both variants, samples homozygous for the variant that is cut by the restriction enzyme was included to provide a background rate of cut failure either due to experimental error or mutation of restriction site during amplification and cloning. The proportion of each allele measured using this approach is consistent with the genotype called from exome SRD data for all samples, except one. The exception is NA12763, where the molecular cloning method generates an estimate which agrees with a copy number of 6 called by PRT rather than a copy number of 4 called by exome SRD, and therefore reflects an error in copy number calling by exome SRD.

We considered whether a signature of selection at these beta-defensin genes could be inferred from the frequency of sequence variants. By comparing the sequence variant frequency distribution of non-synonymous and synonymous SNPs within coding regions, it is possible to detect the effect of negative selection or balancing selection across the region. Assuming that selection does not act on synonymous variants and therefore their variant frequency distribution represents the neutral null model, we would expect to see an enrichment of nonsynonymous variants at low frequency under negative selection, and an enrichment of non-synonymous variants at high frequency (0.4-0.5) under balancing selection.

Given the small exon size and therefore small number of polymorphisms in the coding region of each gene, we compared the sequence variant frequency distribution for each continental group separately, combining data across all beta-defensin genes measured in the CNV. We did not find a statistically significant difference between any non-synonymous and synonymous sequence variant frequency distributions, for any of the continental groups. This suggests that selection is not acting on these genes, and that the sequence variants observed are essentially neutral.

\section{Discussion}

In this study we show that high coverage exome sequencing can effectively report integer copy number and sequence variation between copies. Focusing on exome sequencing data has two disadvantages compared to whole genome sequencing data. Firstly, typing depends on the presence of exons (or, more correctly, baits in the exome enrichment mix) that map within the complex $\mathrm{CNV}$ region. It is obvious that if there are no exons

Table 3 Discrepant copy number calls between exome sequence read depth (SRD) analysis and previous analyses

\begin{tabular}{|c|c|c|c|c|c|c|}
\hline Sample & Pop & Batch & $\begin{array}{l}\text { Posterior probability of } \\
\text { Exome SRD call }\end{array}$ & $\begin{array}{l}\text { Copy number from } \\
\text { Exome SRD }\end{array}$ & $\begin{array}{l}\text { PRT and nanostring } \\
\text { copy number }\end{array}$ & $\begin{array}{l}\text { Copy number from whole } \\
\text { genome SRD }\end{array}$ \\
\hline NA18858 & YRI & BGl & 0.589 & 6 & 8 & 8 \\
\hline NA18861 & YRI & BGl & 0.996 & 5 & 6 & 6 \\
\hline NA11892 & CEU & BGl & 0.968 & 3 & 5 & 3 \\
\hline NA11893 & CEU & BGl & 0.977 & 4 & 5 & 5 \\
\hline NA12156 & CEU & $\mathrm{BCM}$ & 0.610 & 5 & 6 & na \\
\hline NA18912 & YRI & $\mathrm{Bl}$ & 0.982 & 6 & 5 & 6 \\
\hline NA12761 & CEU & WUGSC & 0.995 & 4 & 3 & na \\
\hline
\end{tabular}




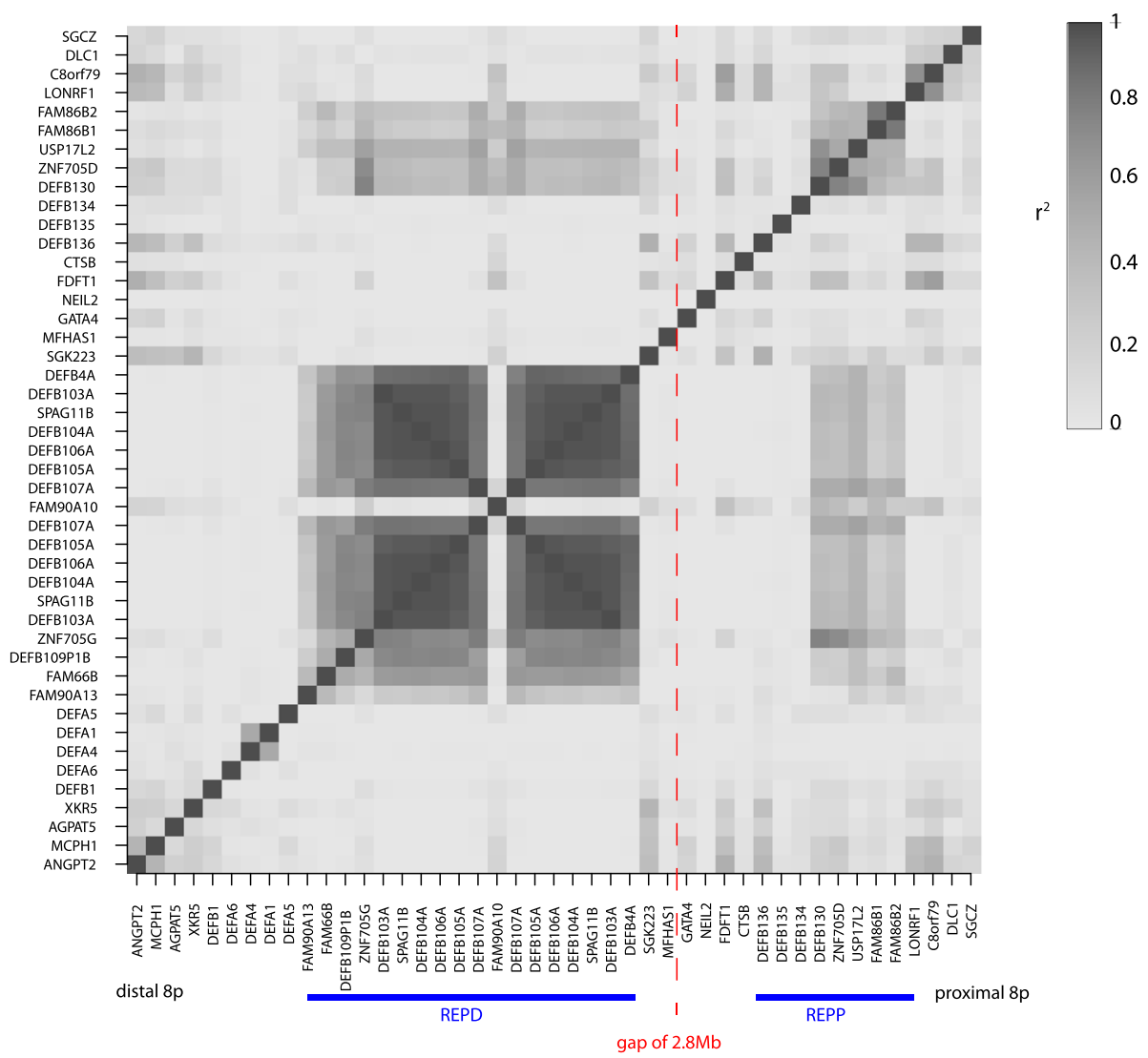

Fig. 5 Correlation of SVD-ZRPKM values between genes at 8p23.1. Plot of pairwise correlation between SVD-ZRPKM values among genes at chromosome region 8p23.1. The SVD-ZRPKM mean for all exons belonging to each gene was calculated and the pairwise correlation for each pair of genes was evaluated by the $r^{2}$ metric (the correlation is increasing with gray shading). Gene presence and location is based on the annotation of the hg19 human genome assembly. Complex repeat-rich regions REPP and REPD are indicated, and several genes between REPP and REPD are omitted to save space, as indicated by the red dashed line

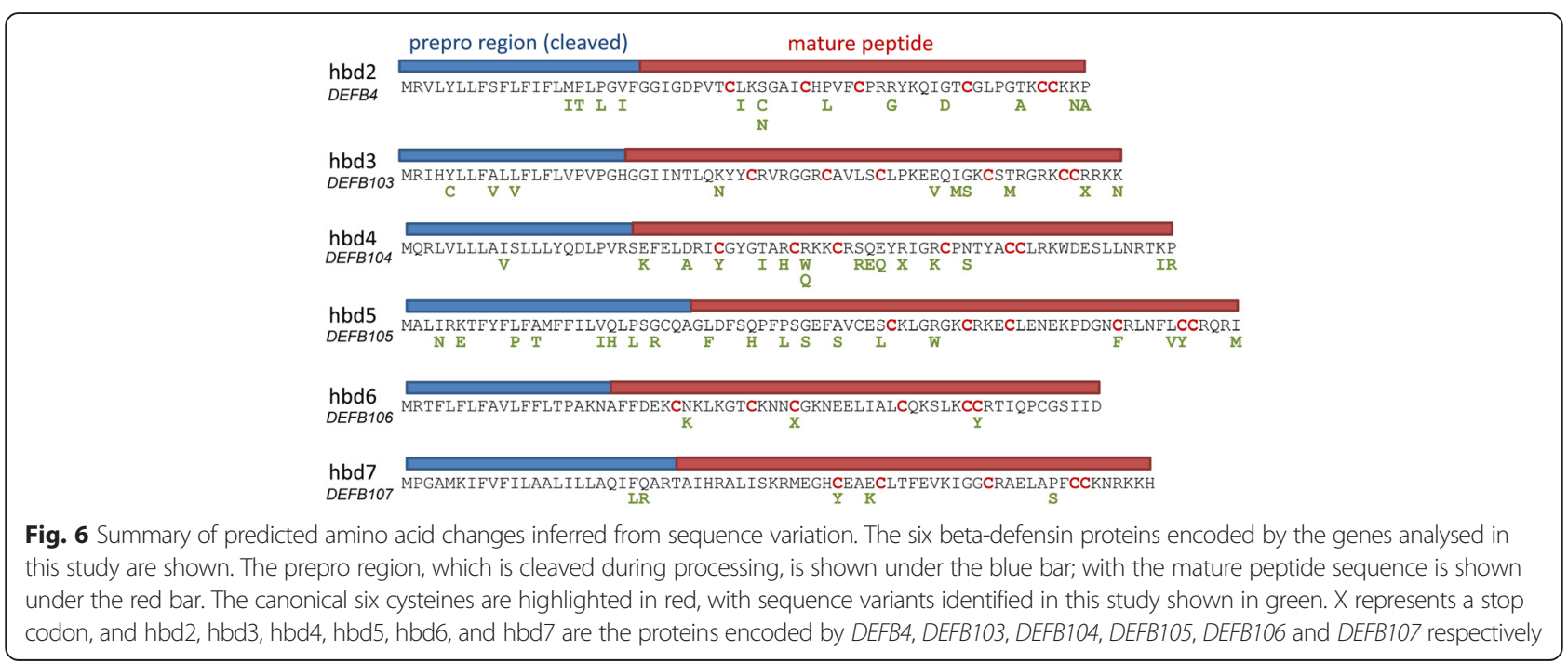


Table 4 Validation of single nucleotide variants

\begin{tabular}{|c|c|c|c|c|c|c|c|c|}
\hline \multirow[t]{2}{*}{ Sample } & \multicolumn{4}{|c|}{ rs140952426 } & \multicolumn{4}{|c|}{ rs 200757797} \\
\hline & Uncut (A) & Cut (G) & Proportion A (95 \% Cl) & From exome & Uncut (T) & Cut $(C)$ & Proportion T $(95 \%$ Cl) & From exome \\
\hline NA07056 & 60 & 41 & $0.59(0.49-0.69)$ & AAGG & 3 & 84 & $0.03(0.01-0.10)$ & CCCC \\
\hline NA12044 & 4 & 74 & $0.05(0.01-0.13)$ & GGGG & 18 & 53 & $0.25(0.16-0.37)$ & $\mathrm{CCCT}$ \\
\hline NA18956 & 44 & 123 & $0.26(0.20-0.34)$ & AGGG & - & - & - & CCCC \\
\hline NA12004 & 37 & 139 & $0.21(0.15-0.28)$ & AGGG & - & - & - & CCCC \\
\hline NA12156 & 13 & 146 & $0.08(0.04-0.14)$ & AGGGG & - & - & - & $\mathrm{CCCCC}$ \\
\hline NA07357 & - & - & - & GGGGGG & 5 & 79 & $0.06(0.02-0.13)$ & СCCCCT \\
\hline NA12763 & - & - & - & GGGG & 22 & 178 & $0.11(0.07-0.16)$ & $\mathrm{CCCT}^{\mathrm{a}}$ \\
\hline NA12874 & - & - & - & GGGG & 16 & 52 & $0.23(0.14-0.35)$ & CCCT \\
\hline
\end{tabular}

$95 \%$ confidence intervals of the binomial distribution calculated using the Pearson-Klopper method

${ }^{\text {a }}$ Called as 6 copies by previous PRT

within the $\mathrm{CNV}$, the sequence will not be enriched and no (or very little) sequence will be generated from that $\mathrm{CNV}$. The problem is scalar, in that it is likely that CNVs with more exonic sequence will have more sequence reads and therefore be more effectively called using SRD approaches compared to CNVs with a small amount of exonic sequence. Secondly, exome sequencing relies on solution-phase enrichment of fragmented DNA using sequence-specific baits. Such an enrichment process might introduce bias into copy number calling, dependent on GC-content of the region or experimental batch, for example.

Future studies need to be aware of several issues that are important to consider when inferring integer copy number from exome SRD. Firstly, in this study we knew a priori the extent of $\mathrm{CNV}$, which had been elucidated previously, so allowing us to choose several genes which we know were contained in a contiguous CNV block. Inferring CNV boundaries from exome data, particularly when those boundaries are within gene sparse areas or segmental duplications, may result in noisier SRD data, although we show that once a CNV can be robustly typed, the boundary of the $\mathrm{CNV}$ can be refined on a gene-by-gene basis. Secondly, we had a number of positive controls where copy number was well established using other methods. This allowed us to validate our singular value decomposition components and our Gaussian mixture models, giving us confidence in our final copy number calls. Thirdly, particular population-sequencing centre batches of samples gave distinct raw copy number results, suggesting that fitting individual Gaussian mixture models to individual batches is important. We do not know what causes this variation, and why sometimes it prevents clustering of raw copy number results, but we suspect it might be due to subtly different signal-noise structures in the data because of different exome enrichment processes used by different centres, which SVD cannot completely resolve. If correct, then we predict that high-coverage whole genome sequences will not show this problem.

One previous study used target enrichment and 454 sequencing of over $87 \mathrm{~kb}$ of the beta-defensin CNV in two samples to study sequence variation [32]. This study had the advantage of revealing variation in non-coding regions, and, together with the longer reads generated by 454 technology, sequence haplotypes could be inferred. However, CNV calling by read depth was not attempted and some sequence variants, particularly rare variants, were called with a small number of supporting reads, suggesting limited sensitivity of the approach. Furthermore, only two European samples were sequenced, revealing a limited part of the total potential sequence variation of the region.

By comparing sequenced BACs, we have previously shown that sequence differences between different copies is localised particularly immediately upstream and downstream of genes, and this may result in expression differences between different copies of the same gene. Indeed, both luciferase reporter studies in keratinocytes and expression in lymphoblastoid cell lines have shown that variation between different defensin copies affects expression of DEFB103 and of DEFB4 [26, 33]. The single nucleotide variation affecting $D E F B 103$ expression is within $1 \mathrm{~kb}$ immediately $5^{\prime}$ of the transcription start site, but the single nucleotide variation affecting DEFB4 expression is not known, except that it is tagged by a synonymous polymorphism rs2740090, which is reported as common across all the continental groups studied here (Additional file 1: Table S1). Our previous observations of increased sequence differentiation upstream of DEFB103, together with its functional effect and the population distribution differences, have led us to suggest that natural selection has influenced expression levels of at least DEFB103 [26]. Furthermore, given the gene dosage effect of copy number variation observed at this locus [34, 35], gene copy 
number results in variation in levels of protein which in turn provides a phenotype upon which selection can act. In contrast, in rhesus macaques [21] and dogs [36] it seems likely that amino acid sequence variation between copies has been subject to natural selection.

In Africa, all variants that alter amino acids in the defensin genes are rare, but outside some of these variants have risen to increased frequency. In particular, a variant at DEFB105 (rs700757797), which disrupts the fifth canonical cysteine essential for disulphide bridge formation in the mature protein (Fig. 6), is common in non-African populations (Additional file 1: Table S1). The functional consequences of this and other variants are not known, and await further study. It is likely that they are variants with little effect or null variants, resulting in proteins with reduced function. In the context of a multicopy gene, one or two copies with little or no function will have little consequence, particularly at higher copy numbers, therefore negative selection against such variants is likely to be weak, and indeed we could not detect any signature of selection using variant frequency distributions. An alternative view is that some variants may have alternative off-target effects, and that we have little power to detect selection at a small number of such variants. Indeed given the known receptor promiscuity of defensins [37] and the influence of structural changes on antimicrobial activity $[38,39]$ this is a possibility worth exploring in the context of novel therapeutic agents.

\section{Conclusions}

We have used exome sequence data from the 1000 Genomes Project to call copy number for the human beta-defensin region on 1285 individuals. We have also identified 436 sequence variants that differ between copies and between individuals, mostly rare, of which 67 are predicted to affect amino-acid sequence of one of the beta-defensin genes.

\section{Additional file}

Additional file 1: Table S1. Frequency of rs 200757797 and rs 2740090 in the 1000 Genomes continental groups. (DOCX $13.4 \mathrm{~kb}$ )

\section{Competing interests}

EJH has received grant funding from Pfizer Inc on a project unrelated to the project described in this paper.

\section{Author contributions}

The work was conceived and designed by DF and EJH. DF and DM generated the data, and DF, EJH, DM, RA and MS analysed and discussed the results. AS provided Nanostring nCounter data. The paper was written by EJH with all authors contributing and reviewing the final manuscript.

\section{Acknowledgements}

This research used the ALICE High Performance Computing Facility at the University of Leicester.

\section{Author details}

'Department of Genetics, University of Leicester, Leicester, UK

${ }^{2}$ Bioinformatics, Scientific Institute IRCCS E.MEDEA, Bosisio, Parini, Italy.

${ }^{3}$ Department of Genetics and Genome Sciences, Icahn School of Medicine at Mount Sinai, New York, NY, USA.

Received: 1 September 2015 Accepted: 22 October 2015

Published online: 02 November 2015

\section{References}

1. Hollox EJ, Hoh B-P: Human gene copy number variation and infectious disease. Hum Genet. 2014;133(10):1217-33.

2. Schrider DR, Hahn MW. Gene copy-number polymorphism in nature. Proc R Soc B Biol Sci. 2010;277(1698):3213-21.

3. Wain LV, Armour JAL, Tobin MD. Genomic copy number variation, human health, and disease. Lancet. 2009;374(9686):340-50.

4. Handsaker RE, Van Doren V, Berman JR, Genovese G, Kashin S, Boettger LM, et al. Large multiallelic copy number variations in humans. Nat Genet. 2015:47(3):296-303.

5. Polley S, Louzada S, Forni D, Sironi M, Balsakas T, Hains D, et al. Evolution of the rapidly-mutating human salivary agglutinin gene (DMBT1) and population subsistence strategy. Proc Natl Acad Sci. 2015;112(15):5105-10.

6. Montavon T, Thevenet L, Duboule D. Impact of copy number variations (CNVs) on long-range gene regulation at the HoxD locus. Proc Natl Acad Sci. 2012;109(50):20204-11.

7. Hollox EJ, Huffmeier U, Zeeuwen PL, Palla R, Lascorz J, Rodijk-Olthuis D, et al. Psoriasis is associated with increased beta-defensin genomic copy number. Nat Genet. 2008:40(1):23.

8. Stuart PE, Hüffmeier U, Nair RP, Palla R, Tejasvi T, Schalkwijk J, et al. Association of $\beta$-defensin copy number and psoriasis in three cohorts of European origin. J Investig Dermatol. 2012;132(10):2407-13.

9. Vittori A, Breda C, Repici M, Orth M, Roos RA, Outeiro TF, et al. Copy number variation of the neuronal glucose transporter gene SLC2A3 and age of onset in Huntington's disease. Hum Mol Genet. 2014;23(12):3129-37.

10. Remnant EJ, Good RT, Schmidt JM, Lumb C, Robin C, Daborn PJ, et al. Gene duplication in the major insecticide target site, Rdl, in Drosophila melanogaster. Proc Natl Acad Sci U S A. 2013;110(36):14705-10.

11. Schmidt JM, Good RT, Appleton B, Sherrard J, Raymant GC, Bogwitz MR, et al. Copy number variation and transposable elements feature in recent, ongoing adaptation at the Cyp6g1 locus. PLoS Genet. 2010;6(6):e1000998.

12. Cantsilieris S, Western PS, Baird PN, White SJ. Technical considerations for genotyping multi-allelic copy number variation (CNV), in regions of segmental duplication. BMC Genomics. 2014;15(1):329.

13. Aldhous MC, Bakar SA, Prescott NJ, Palla R, Soo K, Mansfield JC, et al. Measurement methods and accuracy in copy number variation: failure to replicate associations of beta-defensin copy number with Crohn's disease. Hum Mol Genet. 2010;19(24):4930-8.

14. Fode P, Jespersgaard C, Hardwick RJ, Bogle H, Theisen M, Dodoo D, et al. Determination of beta-defensin genomic copy number in different populations: a comparison of three methods. PLoS One. 2011;6(2):e16768.

15. Sudmant PH, Kitzman JO, Antonacci F, Alkan C, Malig M, Tsalenko A, et al. Diversity of human copy number variation and multicopy genes. Science. 2010;330(6004):641.

16. Tan R, Wang Y, Kleinstein SE, Liu Y, Zhu X, Guo H, et al. An Evaluation of Copy Number Variation Detection Tools from Whole-Exome Sequencing Data. Hum Mutat. 2014;35(7):899-907.

17. Dennis MY, Nuttle X, Sudmant PH, Antonacci F, Graves TA, Nefedov M, et al. Human-specific evolution of novel SRGAP2 genes by incomplete segmental duplication. Cell. 2012;149(4):912.

18. Krumm N, Sudmant PH, Ko A, O'Roak BJ, Malig M, Coe BP, et al. Copy number variation detection and genotyping from exome sequence data. Genome Res. 2012;22(8):1525-32

19. Krumm N, O'Roak BJ, Karakoc E, Mohajeri K, Nelson B, Vives L, et al. Transmission disequilibrium of small CNVs in simplex autism. Am J Hum Genet. 2013:93(4):595-606.

20. Abu Bakar S, Hollox EJ, Armour JA. Allelic recombination between distinct genomic locations generates copy number diversity in human beta-defensins. Proc Natl Acad Sci U S A. 2009;106(3):853-8.

21. Ottolini B, Hornsby MJ, Abujaber R, MacArthur JA, Badge RM, Schwarzacher T, et al. Evidence of convergent evolution in humans and macaques supports an 
adaptive role for copy number variation of the beta-defensin-2 gene. Genome Biol Evol. 2014;6(11):3025-38.

22. Zhou YS, Webb S, Lettice L, Tardif S, Kilanowski F, Tyrrell C, et al. Partial deletion of chromosome 8 beta-defensin cluster confers sperm dysfunction and infertility in male mice. PLoS Genet. 2013;9(10):e1003826.

23. Hollox EJ, Armour JA, Barber JC. Extensive normal copy number variation of a $\beta$-defensin antimicrobial-gene cluster. Am J Hum Genet. 2003;73(3):591-600.

24. Hach F, Sarrafi I, Hormozdiari F, Alkan C, Eichler EE, Sahinalp SC. mrsFASTUltra: a compact, SNP-aware mapper for high performance sequencing applications. Nucleic Acids Res. 2014;42(Web Server issue):W494-500.

25. Hach F, Hormozdiari F, Alkan C, Hormozdiari F, Birol I, Eichler EE, et al. mrsFAST: a cache-oblivious algorithm for short-read mapping. Nat Methods. 2010;7(8):576-7.

26. Hardwick RJ, Machado LR, Zuccherato LW, Antolinos S, Xue Y, Shawa N, et al. A worldwide analysis of beta-defensin copy number variation suggests recent selection of a high-expressing DEFB103 gene copy in East Asia. Hum Mutat. 2011;32(7):743-50.

27. Garrison E, Marth G. Haplotype-based variant detection from short-read sequencing. arXiv preprint. 2012;arXiv:1207-3907. [q-bio.GN] 2012.

28. Brahmachary M, Guilmatre A, Quilez J, Hasson D, Borel C, Warburton P, et al. Digital genotyping of macrosatellites and multicopy genes reveals novel biological functions associated with copy number variation of large tandem repeats. PLoS Genet. 2014;10(6):e1004418.

29. Barnes C, Plagnol V, Fitzgerald T, Redon R, Marchini J, Clayton D, et al. A robust statistical method for case-control association testing with copy number variation. Nat Genet. 2008;40(10):1245-52.

30. Scholz FW, Stephens MA. K-sample Anderson-Darling tests. J Am Stat Assoc. 1987:82(399):918-24.

31. Hollox EJ, Barber JCK, Brookes AJ, Armour JAL. Defensins and the dynamic genome: what we can learn from structural variation at human chromosome band 8p23. 1. Genome Res. 2008;18(11):1686-97.

32. Taudien S, Szafranski K, Felder M, Groth M, Huse K, Raffaelli F, et al. Comprehensive assessment of sequence variation within the copy number variable defensin cluster on 8p23 by target enriched in-depth 454 sequencing. BMC Genomics. 2011;12(1):243.

33. Groth M, Wiegand C, Szafranski K, Huse K, Kramer M, Rosenstiel P, et al. Both copy number and sequence variations affect expression of human DEFB4. Genes Immun. 2010;11(6):458-66.

34. Jansen PA, Rodijk-Olthuis D, Hollox EJ, Kamsteeg M, Tjabringa GS, de Jongh GJ, et al. $\beta$-Defensin-2 protein is a serum biomarker for disease activity in psoriasis and reaches biologically relevant concentrations in lesional skin. PLoS One. 2009;4(3):e4725.

35. Jaradat S, Hoder-Przyrembel C, Cubillos S, Krieg N, Lehmann K, Piehler S, et al. Beta-defensin-2 genomic copy number variation and chronic periodontitis. J Dent Res. 2013;92(11):1035-40.

36. Anderson TM, Candille SI, Musiani M, Greco C, Stahler DR, Smith DW, et al. Molecular and evolutionary history of melanism in North American gray wolves. Science. 2009;323(5919):1339-43.

37. Semple F, Dorin JR. beta-Defensins: multifunctional modulators of infection, inflammation and more? J Innate Immun. 2012;4(4):337-48.

38. Schroeder BO, Wu Z, Nuding S, Groscurth S, Marcinowski M, Beisner J, et al. Reduction of disulphide bonds unmasks potent antimicrobial activity of human beta-defensin 1. Nature. 2011;469(7330):419-23.

39. Wu Z, Hoover DM, Yang D, Boulègue C, Santamaria F, Oppenheim JJ, et al. Engineering disulfide bridges to dissect antimicrobial and chemotactic activities of human $\beta$-defensin 3. Proc Natl Acad Sci. 2003;100(15):8880-5.

\section{Submit your next manuscript to BioMed Central and take full advantage of:}

- Convenient online submission

- Thorough peer review

- No space constraints or color figure charges

- Immediate publication on acceptance

- Inclusion in PubMed, CAS, Scopus and Google Scholar

- Research which is freely available for redistribution 